\title{
$A L$-空间单位球面上的等距算子的延拓
}

定光桂

南开大学数学科学学院, 核心数学与组合数学重点实验室, 天津 300071

E-mail: ding_gg@nankai.edu.cn

收稿日期: 2007-09-08; 接受日期: 2007-09-19

国家自然科学基金 (批准号: 10571090) 和高校博士点基金 (批准号: 20060055010) 资助项目

摘要证明了 $A L$ - 空间和 Banach 空间单位球面之间的满等距算子均可以延拓为全空间 上的线性等距算子.

关键词重分形谱 等距延拓 (原子) $A L$ - 空间

$\operatorname{MSC}(2000)$ 主题分类 $46 \mathrm{A22}, 46 \mathrm{B02}, 46 \mathrm{B20}$

\section{1 简介}

1987 年, Tingley ${ }^{[1]}$ 曾提出关于单位球面间的等距算子的延拓问题如下: 令 $E$ 和 $F$ 为实 Banach 空间. 设 $V_{0}$ 是单位球面 $S_{1}(E)$ 和 $S_{1}(F)$ 间的满等距算子, 则 $V_{0}$ 是否一定为 $E$ 上的 线性或者仿射等距算子在 $S_{1}(E)$ 上的限制?

过去 10 年来, 我和我的学生们一直在研究这个专题, 得到了许多重要的结果. 特别地, 对 于一系列经典的 Banach 空间, 如 $\ell^{p}(\Gamma), L^{p}(\mu)$, 更一般地, $A L^{p}$ 空间 $(1 \leqslant p<\infty)$ 和 $C(\Omega)$; 以及一些完备赋准范空间 $\ell^{\beta}(\Gamma), L^{\beta}(\mu)(0<\beta<1),\left(\ell^{\beta_{n}}\right)$ 和 $(s)$ (例见文献 [2-8]), 我们均获 得了关于 Tingley 问题的肯定答案. 上面提到的文章几乎都只考虑同一类型空间之间的单位 球面的满等距算子. 在文献 [9] 中, 作者首次讨论了两种不同类型空间之间的等距算子, 并获 得肯定答案. 并且作者最先考虑非满等距算子的延拓问题, 并在文献 [10] 中, 作者得到了关 于内积空间的肯定结果.

在文献 [5] 中, 王建提出在某些条件下, “原子” $A L^{p}$ - 空间 $(0<p<\infty, p \neq 2)$ 的单位球 面间的非满等距算子可以线性等距延拓, 后来在文献 [11] 中对于 $1<p<\infty$ 情形, 侯志涁不 加条件地证明了同样的结论.

接下来, 我们开始考虑不同类型 Banach 空间之间 (从 $S_{1}\left(\ell_{(2)}^{\infty}\right)$ 到 $S_{1}\left(L^{1}(\mu)\right.$ ) 内, 及从 $S_{1}(\ell(\Gamma))$ 或 $S_{1}\left(\mathcal{L}^{\infty}(\Gamma)\right)$ 到 $S_{1}(E)$ 内, 其中 $E$ 为 Banach 空间) 的非满等距算子的 Tingley 等 距延拓问题, 文献 [12] 及 [13] 都得到了一些有用的结果. 另外文献 [14] (等距算子从 $S_{1}(E)$ 到 $S_{1}(C[0,1])$ 上) 和文献 [15] (等距算子从 $S_{1}(E)$ 到 $S_{1}\left(\ell^{\infty}(\Gamma)\right)$ 内) 均肯定或部分肯定回答了等 
距算子的延拓问题 (其中 $E$ 为 Banach 空间). 文献 [16] 对于 $\mathcal{L}^{\infty}$ - 类型空间和某些 Banach 空 间的单位球面之间的等距延拓问题也部分地做出了肯定回答.

本文是文献 [17] 的拓展, 文中推论 8 、定理 13 和推论 14 推广了文献 [17] 中的一些主要结 果. 由于用 $L(T, \mu)$ 来取代 $\ell(\Gamma)$ 研究上述的延拓问题是非常困难的, 故必须用一些与文献 [17] 极其不同的技巧. 本文将指出两个 $A L$-空间的单位球面之间的等距算子能够线性等距延拓的 充要条件. 特别地, 指出了从原子 $A L$-空间的单位球面到另一 “原子 $A L$-空间” 的单位球面内, 更一般地, 到 $A L$-空间的单位球面内的等距算子的线性等距延拓条件. 注意到 Kakutani 的表 现定理 (文献 [18], 定理 1.b.2), 我们只需考虑 $L(T, \mu)$ 空间上的延拓问题, 其中 $T$ 是 Hausdorff 空间, $\mu$ 是 $T$ 上 Baire 测度.

本文涉及到的空间都是实数域上的线性空间.

\section{2 引理}

引理 1 设 $E$ 是 Banach 空间, $V_{0}$ 是从单位球面 $S_{1}(L(T, \mu))$ 到单位球面 $S_{1}(E)$ 内 的等距算子. 如果有 $-V_{0}\left[S_{1}(L(T, \mu))\right] \subset V_{0}\left[S_{1}(L(T, \mu))\right]$, 那么, 对于 $T$ 中每一个可测子集 $A(0<\mu(A)<\infty)$, 均有

$$
V_{0}\left(-\frac{\chi_{A}}{\mu(A)}\right)=-V_{0}\left(\frac{\chi_{A}}{\mu(A)}\right)
$$

其中, $\chi_{A}$ 是 $A$ 的特征函数.

证明 由于证明较长, 下面分三步进行证明:

步骤 I 从假设可知, 存在 $x \in S_{1}(L(T, \mu))$, 使得

$$
V_{0}(x)=-V_{0}\left(\frac{\chi_{A}}{\mu(A)}\right) \text {. }
$$

令

$$
A_{1}^{+}=\left\{t: 0 \leqslant x(t) \leqslant \frac{1}{\mu(A)}, t \in A\right\}, \quad A_{2}^{+}=\left\{t: x(t)>\frac{1}{\mu(A)}, t \in A\right\},
$$

和

$$
A_{1}^{-}=\left\{t:-\frac{1}{\mu(A)} \leqslant x(t) \leqslant 0, t \in A\right\}, \quad A_{2}^{-}=\left\{t: x(t)<-\frac{1}{\mu(A)}, t \in A\right\} .
$$

然后令 $A^{+}=A_{1}^{+} \cup A_{2}^{+}$和 $A^{-}=A_{1}^{-} \cup A_{2}^{-}$.

由 $V_{0}$ 的假设和 (1) 式, 有

$$
\begin{aligned}
2= & \left\|V_{0}(x)-V_{0}\left(\frac{\chi_{A}}{\mu(A)}\right)\right\|=\left\|x-\frac{\chi_{A}}{\mu(A)}\right\| \\
= & \int_{A}\left|x(t)-\frac{1}{\mu(A)}\right| \mu(d t)+\int_{A^{c}}|x(t)| \mu(d t) \\
= & \int_{A_{1}^{+}}\left(\frac{1}{\mu(A)}-|x(t)|\right) \mu(d t)+\int_{A_{2}^{+}}\left(|x(t)|-\frac{1}{\mu(A)}\right) \mu(d t) \\
& +\int_{A^{-}}\left(|x(t)|+\frac{1}{\mu(A)}\right) \mu(d t)+\int_{A^{c}}|x(t)| \mu(d t) .
\end{aligned}
$$

又由 $\|x\|=1$ 和 $\int_{A} \frac{1}{\mu(A)} \mu(d t)=1$, 从 (2) 式则可导出

$$
2=1-2 \int_{A_{2}^{+}} \frac{1}{\mu(A)} \mu(d t)+1-2 \int_{A_{1}^{+}}|x(t)| \mu(d t),
$$


也即

$$
\int_{A_{1}^{+}}|x(t)| \mu(d t)=-\int_{A_{2}^{+}} \frac{1}{\mu(A)} \mu(d t) .
$$

因此, 由 (3) 式, 我们得到 $\mu\left(A_{2}^{+}\right)=0$ 和 $x(t)=0$ (a.e. $t \in A_{1}^{+}$). 即有

$$
\operatorname{supp} x \subset A^{-} \cup A^{c} .
$$

步骤 II 同样从假设可知, 对于每一个可测子集 $B \subset T$, 满足 $B \cap A=\emptyset(0<\mu(B)<\infty)$, 均存在两个元 $y_{ \pm} \in S_{1}(L(T, \mu))$, 使得

$$
V_{0}\left(y_{ \pm}\right)=-V_{0}\left(\frac{1}{2} \frac{\chi_{A}}{\mu(A)} \pm \frac{1}{2} \frac{\chi_{B}}{\mu(B)}\right),
$$

从而有

$$
\begin{aligned}
2 & =\left\|V_{0}\left(y_{ \pm}\right)-V_{0}\left(\frac{1}{2} \frac{\chi_{A}}{\mu(A)} \pm \frac{1}{2} \frac{\chi_{B}}{\mu(B)}\right)\right\| \\
& =\left\|y_{ \pm}-\left(\frac{1}{2} \frac{\chi_{A}}{\mu(A)} \pm \frac{1}{2} \frac{\chi_{B}}{\mu(B)}\right)\right\| \\
& =\int_{A}\left|y_{ \pm}(t)-\frac{1}{2 \mu(A)}\right| \mu(d t)+\int_{B}\left|y_{ \pm}(t) \mp \frac{1}{2 \mu(B)}\right| \mu(d t)+\int_{(A \cup B)^{c}}\left|y_{ \pm}(t)\right| \mu(d t) .
\end{aligned}
$$

注意到

$$
\left\|y_{ \pm}\right\|=\int_{T}\left|y_{ \pm}(t)\right| \mu(d t)=1, \quad \int_{A} \frac{1}{2 \mu(A)} \mu(d t)=\int_{B} \frac{1}{2 \mu(B)} \mu(d t)=\frac{1}{2},
$$

故从 (6) 式我们可以导出

$$
y_{ \pm}(t) \leqslant 0, \quad \text { a.e. } \quad t \in A,
$$

和

$$
y_{+}(t) \leqslant 0, \quad y_{-}(t) \geqslant 0, \quad \text { a.e. } \quad t \in B,
$$

且由 (7) 式立即可以得到

$$
\begin{aligned}
\left\|y_{ \pm}-\frac{\chi_{A}}{\mu(A)}\right\| & =\int_{A}\left|y_{ \pm}(t)-\frac{1}{\mu(A)}\right| \mu(d t)+\int_{A^{c}}\left|y_{ \pm}(t)\right| \mu(d t) \\
& =\int_{A}\left(\left|y_{ \pm}(t)\right|+\frac{1}{\mu(A)}\right) \mu(d t)+\int_{A^{c}}\left|y_{ \pm}(t)\right| \mu(d t)=2 .
\end{aligned}
$$

由于 $V_{0}$ 是等距算子, 通过 (1) 和 (5) 式, 则有

$$
\begin{aligned}
2 & =\left\|V_{0}\left(y_{ \pm}\right)-V_{0}\left(\frac{\chi_{A}}{\mu(A)}\right)\right\| \\
& =\left\|-V_{0}\left(\frac{1}{2} \frac{\chi_{A}}{\mu(A)} \pm \frac{1}{2} \frac{\chi_{B}}{\mu(B)}\right)+V_{0}(x)\right\| \\
& =\left\|x-\left(\frac{1}{2} \frac{\chi_{A}}{\mu(A)} \pm \frac{1}{2} \frac{\chi_{B}}{\mu(B)}\right)\right\| \\
& =\int_{A}\left|x(t)-\frac{1}{2 \mu(A)}\right| \mu(d t)+\int_{B}\left|x(t) \mp \frac{1}{2 \mu(B)}\right| \mu(d t)+\int_{(A \cup B)^{c}}|x(t)| \mu(d t) .
\end{aligned}
$$

结合 (4) 式和 $\|x\|=1$, 由上面等式则可导出

$$
2=\int_{A}|x(t)| \mu(d t)+\frac{1}{2}+\int_{B}\left|x(t) \mp \frac{1}{2 \mu(B)}\right| \mu(d t)+\int_{(A \cup B)^{c}}|x(t)| \mu(d t)
$$




$$
=\left(1-\int_{B}|x(t)| \mu(d t)\right)+\frac{1}{2}+\int_{B}\left|x(t) \mp \frac{1}{2 \mu(B)}\right| \mu(d t) .
$$

也即有

$$
\int_{B}\left(\left|x(t) \mp \frac{1}{2 \mu(B)}\right|-|x(t)|\right) \mu(d t)=\frac{1}{2} .
$$

注意到可测集 $B$ 的任意性, 因此由 (9) 式, 可以得到

$$
x(t)=0, \quad \text { a.e. } \quad t \in A^{c},
$$

且由 (4) 和 (10) 式可导出

$$
\operatorname{supp} x \subset A^{-} .
$$

步骤 III 下面证明 $x(t)$ 在 $A^{-}$内是常值函数. 事实上, 由引理 1 的假设, 对于每一个测 度有限且非零的可测集 $A_{0} \subset A$, 存在 $x_{0} \in S_{1}(L(T, \mu))$, 使得

$$
V_{0}\left(x_{0}\right)=-V_{0}\left(\frac{\chi_{A_{0}}}{\mu\left(A_{0}\right)}\right) \text {. }
$$

类似于上面的步骤 II, 可得

$$
\operatorname{supp} x_{0} \subset A_{0}^{-} \text {. }
$$

由 $\left\|x_{0}\right\|=1$ 和 $(11)$ 式, 有

$$
\begin{aligned}
\left\|x-x_{0}\right\| & =\int_{A \backslash A_{0}}|x(t)| \mu(d t)+\int_{A_{0}}\left|x(t)-x_{0}(t)\right| \mu(d t) \\
& \geqslant \int_{A \backslash A_{0}}|x(t)| \mu(d t)+\int_{A_{0}}\left|x_{0}(t)\right| \mu(d t)-\int_{A_{0}}|x(t)| \mu(d t) \\
& =\left(1-\int_{A_{0}}|x(t)| \mu(d t)\right)+1-\int_{A_{0}}|x(t)| \mu(d t) \\
& =2\left(1-\int_{A_{0}}|x(t)| \mu(d t)\right) .
\end{aligned}
$$

另一方面, 从 (1) 和 (12) 式, 又有

$$
\begin{aligned}
\left\|V_{0}(x)-V_{0}\left(x_{0}\right)\right\| & =\left\|-V_{0}\left(\frac{\chi_{A}}{\mu(A)}\right)+V_{0}\left(\frac{\chi_{A_{0}}}{\mu\left(A_{0}\right)}\right)\right\| \\
& =\left\|\frac{\chi_{A_{0}}}{\mu\left(A_{0}\right)}-\frac{\chi_{A}}{\mu(A)}\right\|=2\left(1-\frac{\mu\left(A_{0}\right)}{\mu(A)}\right) .
\end{aligned}
$$

因此从 (13) 和 (14) 式, 我们导出

$$
\int_{A_{0}}|x(t)| \mu(d t) \geqslant \frac{\mu\left(A_{0}\right)}{\mu(A)},
$$

即

$$
\int_{A_{0}}\left(|x(t)|-\frac{1}{\mu(A)}\right) \mu(d t) \geqslant 0 .
$$

注意到子集 $A_{0} \subset A$ 的任意性, 从上则有

$$
|x(t)| \geqslant \frac{1}{\mu(A)}, \quad \text { a.e. } \quad t \in A .
$$

因此, 由于 $\|x\|=1$, 结合 (11) 和 (15) 式, 立即得到

$$
x=-\frac{\chi_{A}}{\mu(A)} \text {. }
$$


证毕.

下面的结论类似于文献 [2] 中的引理 3.

引理 2 在引理 1 的假设条件下, 如果 $E$ 为空间 $L(S, \nu)$, 那么, 对于任意的 $x \in S_{1}(L(T, \mu))$, 均有

$$
\mu(\operatorname{supp} x \cap A)=0 \quad \Longleftrightarrow \quad \nu\left(\operatorname{supp} V_{0}(x) \cap \operatorname{supp} V_{0}\left(\frac{\chi_{A}}{\mu(A)}\right)\right)=0 .
$$

特别地,

$$
\mu\left(A_{1} \cap A_{2}\right)=0 \quad \Longleftrightarrow \quad \nu\left(\operatorname{supp} V_{0}\left(\frac{\chi_{A_{1}}}{\mu\left(A_{1}\right)}\right) \cap \operatorname{supp} V_{0}\left(\frac{\chi_{A_{2}}}{\mu\left(A_{2}\right)}\right)\right)=0
$$

(其中, $A, A_{1}$ 和 $A_{2}$ 均是 $T$ 中测度有限且非零的可测子集).

证明 由 $V_{0}$ 的假设及引理 1 , 我们有

$$
\begin{aligned}
\left\|V_{0}(x) \pm V_{0}\left(\frac{\chi_{A}}{\mu(A)}\right)\right\| & =\left\|x \pm \frac{\chi_{A}}{\mu(A)}\right\| \\
& =\|x\|+\left\|\frac{\chi_{A}}{\mu(A)}\right\|=2 \\
& =\left\|V_{0}(x)\right\|+\left\|V_{0}\left(\frac{\chi_{A}}{\mu(A)}\right)\right\|,
\end{aligned}
$$

从而容易证得本结论.

下面的引理是文献 [17] 中的引理 5 .

引理 3 设 $E$ 为 Banach 空间, $\left\{y_{1}, y_{2}, \ldots, y_{n}\right\}$ 为单位球面 $S_{1}(E)$ 中任意 $n$ 个元. 对于 任意的 $n$ 个数 $\theta_{k}= \pm 1(1 \leqslant k \leqslant n)$, 如其满足

$$
\left\|\theta_{1} y_{1}+\theta_{2} y_{2}+\cdots+\theta_{n} y_{n}\right\|=n
$$

则有

$$
\left\|\sum_{k=1}^{n} \xi_{k} y_{k}\right\|=\sum_{k=1}^{n}\left|\xi_{k}\right|, \quad \forall \xi_{k} \in \mathbb{R}(1 \leqslant k \leqslant n) .
$$

引理 4 在引理 1 的假设条件下, 对于 $T$ 中任意 $n$ 个相互不交的可测子集 $\left\{A_{1}, A_{2}, \ldots, A_{n}\right\}$, 其中 $0<\mu\left(A_{i}\right)<\infty(i=1,2, \ldots, n)$, 如果满足下列条件:

(i) 对任意的数 $\theta_{k}= \pm 1(1 \leqslant k \leqslant n)$, 均有

$$
\left\|\sum_{k=1}^{n} \theta_{k} V_{0}\left(\frac{\chi_{A_{k}}}{\mu\left(A_{k}\right)}\right)\right\|=n,
$$

(ii) 如果有

$$
V_{0}(x)=\sum_{k=1}^{n} \xi_{k} V_{0}\left(\frac{\chi_{A_{k}}}{\mu\left(A_{k}\right)}\right)
$$

就有

$$
x=\sum_{k=1}^{n} \xi_{k}^{\prime} \frac{\chi_{A_{k}}}{\mu\left(A_{k}\right)}+x_{0}
$$

(其中 $\left.\operatorname{supp} x_{0} \subset\left(\bigcup_{k=1}^{n} A_{k}\right)^{c}\right)$.

那么, 必有 $\xi_{k}^{\prime}=\xi_{k}(1 \leqslant k \leqslant n)$ 和 $x_{0}=\theta$.

证明 不失一般性, 我们只需验证 $\xi_{1}(\neq 0)$. 
事实上, 由于 $\|x\|=1$, 从引理 3 和条件 (ii), 有

$$
\begin{aligned}
\left\|V_{0}(x)-\frac{\xi_{1}}{\left|\xi_{1}\right|} V_{0}\left(\frac{\chi_{A_{1}}}{\mu\left(A_{1}\right)}\right)\right\| & =\left\|\sum_{k=1}^{n} \xi_{k} V_{0}\left(\frac{\chi_{A_{k}}}{\mu\left(A_{k}\right)}\right)-\frac{\xi_{1}}{\left|\xi_{1}\right|} V_{0}\left(\frac{\chi_{A_{1}}}{\mu\left(A_{1}\right)}\right)\right\| \\
& =\left\|\sum_{k=2}^{n} \xi_{k} V_{0}\left(\frac{\chi_{A_{k}}}{\mu\left(A_{k}\right)}\right)+\left(\xi_{1}-\frac{\xi_{1}}{\left|\xi_{1}\right|}\right) V_{0}\left(\frac{\chi_{A_{1}}}{\mu\left(A_{1}\right)}\right)\right\| \\
& =\sum_{k=2}^{n}\left|\xi_{k}\right|+\left|\xi_{1}-\frac{\xi_{1}}{\left|\xi_{1}\right|}\right| \\
& =\left(1-\left|\xi_{1}\right|\right)+|1-| \xi_{1}||=2\left(1-\left|\xi_{1}\right|\right) .
\end{aligned}
$$

由于 $V_{0}$ 是等距算子且 $\|x\|=1$, 应用引理 1 , 继续上面等式如下:

$$
\begin{aligned}
& =\left\|x-\frac{\xi_{1}}{\left|\xi_{1}\right|} \frac{\chi_{A_{1}}}{\mu\left(A_{1}\right)}\right\| \\
& =\left\|\sum_{k=1}^{n} \xi_{k}^{\prime} \frac{\chi_{A_{k}}}{\mu\left(A_{k}\right)}+x_{0}-\frac{\xi_{1}}{\left|\xi_{1}\right|} \frac{\chi_{A_{1}}}{\mu\left(A_{1}\right)}\right\| \\
& =\left|\xi_{1}^{\prime}-\frac{\xi_{1}}{\left|\xi_{1}\right|}\right|+\sum_{k=2}^{n}\left|\xi_{k}^{\prime}\right|+\left\|x_{0}\right\| \\
& =\left(1-\frac{\xi_{1}}{\left|\xi_{1}\right|} \xi_{1}^{\prime}\right)+\left(1-\left|\xi_{1}^{\prime}\right|\right)=2-\left|\xi_{1}^{\prime}\right|-\frac{\xi_{1}}{\left|\xi_{1}\right|} \xi_{1}^{\prime} .
\end{aligned}
$$

结合 (16) 和 (17) 式, 可导出

$$
\left|\xi_{1}^{\prime}\right|+\frac{\xi_{1}}{\left|\xi_{1}\right|} \xi_{1}^{\prime}=2\left|\xi_{1}\right|
$$

因此我们便可得到 $\xi_{1}^{\prime}=\xi_{1}$. 类似地, 我们亦可得到 $\xi_{k}^{\prime}=\xi_{k}(2 \leqslant k \leqslant n)$. 最后注意到 $\|x\|=1$ 和 $x_{0}$ 的定义, 从前面的结果立即导出 $x_{0}=\theta$. 这样我们就完成了引理的证明.

\section{3 主要结果}

定理 5 设 $E$ 是 Banach 空间, $V_{0}$ 是从单位球面 $S_{1}(L(T, \mu))$ 到单位球面 $S_{1}(E)$ 内的等 距算子, 则 $V_{0}$ 能够延拓成全空间 $L(T, \mu)$ 上的 (实) 线性等距算子的充分必要条件是:

(i) 对于任意的 $x_{1}, x_{2} \in S_{1}(L(T, \mu))$ 和 $\xi_{1}, \xi_{2} \in \mathbb{R}$, 有

$$
\left\|\xi_{1} V_{0}\left(x_{1}\right)+\xi_{2} V_{0}\left(x_{2}\right)\right\|=1 \Longrightarrow \xi_{1} V_{0}\left(x_{1}\right)+\xi_{2} V_{0}\left(x_{2}\right) \in V_{0}[S(L(T, \mu))] .
$$

(ii) 对于 $T$ 中任意 $n$ 个相互不交可测子集 $\left\{A_{1}, A_{2}, \ldots, A_{n}\right\}$, 其中 $0<\mu\left(A_{i}\right)<\infty(i=$ $1,2, \ldots, n)$, 如果有

$$
V_{0}(x)=\sum_{k=1}^{n} \xi_{k} V_{0}\left(\frac{\chi_{A_{k}}}{\mu\left(A_{k}\right)}\right)
$$

就有

$$
x=\sum_{k=1}^{n} \xi_{k}^{\prime} \frac{\chi_{A_{k}}}{\mu\left(A_{k}\right)}+x_{0}
$$

(这里 $\left.\operatorname{supp} x_{0} \subset\left(\bigcup_{k=1}^{n} A_{k}\right)^{c}\right)$.

证明 必要性是显然的.

下面证明充分性. 设条件 (i) 和 (ii) 成立. 
首先断言对于任意 $n$ 个元 $\left\{x_{1}, x_{2}, \ldots, x_{n}\right\} \subset S_{1}(L(T, \mu))$ 及 $n$ 个实数 $\left\{\xi_{1}, \xi_{2}, \ldots, \xi_{n}\right\}$, 必 有

$$
\left\|\sum_{k=1}^{n} \xi_{k} V_{0}\left(x_{k}\right)\right\|=1 \Longrightarrow \sum_{k=1}^{n} \xi_{k} V_{0}\left(x_{k}\right) \in V_{0}\left[S_{1}(L(T, \mu))\right] .
$$

事实上, 当 $n=2$ 时, 从条件 (i) 可知 (18) 式成立. 假设 $n=m-1$ 时, 有 (18) 式成立, 则当注 意到关系式

$$
\sum_{k=1}^{m} \xi_{k} V_{0}\left(x_{k}\right)=\left\|\sum_{i=1}^{m-1} \xi_{i} V_{0}\left(x_{i}\right)\right\| \sum_{k=1}^{m-1} \frac{\xi_{k}}{\left\|\sum_{i=1}^{m-1} \xi_{i} V_{0}\left(x_{i}\right)\right\|} V_{0}\left(x_{k}\right)+\xi_{m} V_{0}\left(x_{m}\right),
$$

则由归纳假设容易证得断言.

其次, 我们证明对于任意 $n$ 个支撑相互不交的特征函数 $\left\{\chi_{A_{1}}, \chi_{A_{2}}, \ldots, \chi_{A_{n}}\right\}$ (其中 $A_{k} \subset$ $\left.T, 0<\mu\left(A_{k}\right)<\infty\right)$ 和 $\theta_{k}= \pm 1(1 \leqslant k \leqslant n)$, 均有

$$
\left\|\sum_{k=1}^{n} \theta_{k} V_{0}\left(\frac{\chi_{A_{k}}}{\mu\left(A_{k}\right)}\right)\right\|=n .
$$

同样地, 我们可以由归纳法得到 (20) 式. 事实上, 当 $n=2$ 时, 由 $V_{0}$ 是等距算子及引理 1 容易验证 (20) 式成立. 当设 $n=m-1$ 时, $(20)$ 式成立, 则存在 $\hat{x} \in S_{1}(L(T, \mu))$, 使得

$$
V_{0} \hat{x}=\frac{\sum_{k=1}^{m-1} \theta_{k} V_{0}\left(\frac{\chi_{A_{k}}}{\mu\left(A_{k}\right)}\right)}{m-1} .
$$

反之, 如设当 $n=m$ 时, (20) 式不成立. 假设有

则由引理 1 , 可以得到

$$
\left\|\sum_{k=1}^{m} \theta_{k} V_{0}\left(\frac{\chi_{A_{k}}}{\mu\left(A_{k}\right)}\right)\right\|<m
$$

$$
\begin{aligned}
\left\|\hat{x}+\theta_{m} \frac{\chi_{A_{m}}}{\mu\left(A_{m}\right)}\right\| & =\left\|V_{0} \hat{x}+\theta_{m} V_{0}\left(\frac{\chi_{A_{m}}}{\mu\left(A_{m}\right)}\right)\right\| \\
& =\left\|\frac{\sum_{k=1}^{m} \theta_{k} V_{0}\left(\frac{\chi_{A_{k}}}{\mu\left(A_{k}\right)}\right)}{m-1}+\frac{m-2}{m-1} \theta_{m} V_{0}\left(\frac{\chi_{A_{m}}}{\mu\left(A_{m}\right)}\right)\right\| \\
& \leqslant\left\|\frac{\sum_{k=1}^{m} \theta_{k} V_{0}\left(\frac{\chi_{A_{k}}}{\mu\left(A_{k}\right)}\right)}{m-1}\right\|+\left\|\frac{m-2}{m-1} \theta_{m} V_{0}\left(\frac{\chi_{A_{m}}}{\mu\left(A_{m}\right)}\right)\right\|<2 .
\end{aligned}
$$

另一方面, 由引理 4, 从归纳假设和 $(21)$ 式可以导出

$$
\hat{x}=\frac{\sum_{k=1}^{m-1} \theta_{k} \frac{\chi_{A_{k}}}{\mu\left(A_{k}\right)}}{m-1},
$$

因此又有

$$
\begin{aligned}
\left\|\hat{x}+\theta_{m} \frac{\chi_{A_{m}}}{\mu\left(A_{m}\right)}\right\| & =\left\|\frac{\sum_{k=1}^{m-1} \theta_{k} \frac{\chi_{A_{k}}}{\mu\left(A_{k}\right)}}{m-1}+\theta_{m} \frac{\chi_{A_{m}}}{\mu\left(A_{m}\right)}\right\| \\
& =\frac{m-1}{m-1}+1=2 .
\end{aligned}
$$

由于 (22) 和 (24) 式显然是矛盾的, 故证得 (20) 式的结论.

这样一来, 我们从 (20) 式和条件 (ii) 及引理 4 立即导出, 对于 $T$ 中任意 $n$ 个测度有限且 非零的相互不交的可测子集 $\left\{A_{1}, A_{2}, \ldots, A_{n}\right\}$ 和 $\left\{\xi_{1}^{0}, \xi_{2}^{0}, \ldots, \xi_{n}^{0}\right\} \subset \mathbb{R}$, 且 $\sum_{k=1}^{n}\left|\xi_{k}^{0}\right|=1$, 必有 以下关系式:

$$
V_{0}\left(\sum_{k=1}^{n} \xi_{k}^{0} \frac{\chi_{A_{k}}}{\mu\left(A_{k}\right)}\right)=\sum_{k=1}^{n} \xi_{k}^{0} V_{0}\left(\frac{\chi_{A_{k}}}{\mu\left(A_{k}\right)}\right)
$$


也即 $V_{0}$ 限制在单位球面 $S_{1}(L(T, \mu))$ 的简单函数的集合上是线性的.

最后, 当令 $X$ 为空间 $L(T, \mu)$ 内所有简单函数的全体, 并定义

$$
V_{1} x=V_{1}\left(\sum_{k=1}^{n} \lambda_{k} \frac{\chi_{A_{k}}}{\mu\left(A_{k}\right)}\right)=\sum_{k=1}^{n} \lambda_{k} V_{0}\left(\frac{\chi_{A_{k}}}{\mu\left(A_{k}\right)}\right), \quad \forall x=\sum_{k=1}^{n} \lambda_{k} \frac{\chi_{A_{k}}}{\mu\left(A_{k}\right)} \in X(\subset L(T, \mu)),
$$

其中 $\left\{A_{1}, A_{2}, \ldots, A_{n}\right\}$ 是 $T$ 中任意 $n$ 个相互不交的可测子集, 且 $0<\mu\left(A_{i}\right)<\infty(1 \leqslant i \leqslant n)$. 则由引理 3 和 $(20)$ 式, 有

$$
\left\|V_{1} x\right\|=\sum_{k=1}^{n}\left|\lambda_{k}\right|=\|x\|, \quad \forall x=\sum_{k=1}^{n} \lambda_{k} \frac{\chi_{A_{k}}}{\mu\left(A_{k}\right)} \in X .
$$

也就是说, 我们已将 $V_{0}$ 线性等距地延拓到 $L(T, \mu)$ 的子空间 $X$ 上. 而当注意到对于任意 $x \in L(T, \mu)$, 均有 $\mu(\{t:|x(t)|>\lambda, t \in T\})<\infty(\forall \lambda>0)$, 因此我们可以看出 $X$ 是稠于空间 $L(T, \mu)$ 的. 最后由于 $V_{1}$ 是 $X$ 上的等距算子以及 $L(T, \mu)$ 和 $E$ 的完备性, 立即可以导出等距 算子 $V_{1}$ 能唯一延拓成全空间 $L(T, \mu)$ 上的线性等距算子 $V$, 因此 $V$ 就是所求的 $V_{0}$ 的线性等 距延拓算子. 定理证毕.

注 6 从定理 5 的第 2 部分证明过程可得, 对于任意 $x \in S_{1}(L(T, \mu))$, 如果满足

$$
V_{0}(-x)=-V_{0}(x),
$$

则当将特征函数组 $\left\{\frac{\chi_{A_{1}}}{\mu\left(A_{1}\right)}, \frac{\chi_{A_{2}}}{\mu\left(A_{2}\right)}, \ldots, \frac{\chi_{A_{n}}}{\mu\left(A_{n}\right)}\right\}$ 换成相互不交的元组 $\left\{x_{1}, x_{2}, \ldots, x_{n}\right\} \subset S_{1}(L(T$, $\mu))$ 时, 类似的引理 4 同样成立, 因此在这种情形下 (20) 式也是成立的, 更进一步, 定理 5 也 是正确的.

特别地, 如果 $L(T, \mu)$ 是原子生成的, 那么我们有下面简单的推论:

推理 7 设 $E$ 是 Banach 空间, $V_{0}$ 是从单位球面 $S_{1}(\ell(\Gamma))$ 到单位球面 $S_{1}(E)$ 内的等距 算子, 则 $V_{0}$ 能够延拓成全空间 $\ell(\Gamma)$ 上的线性等距算子的充要条件是: 对于任意两个支撑不 交的元 $x_{1}, x_{2} \in S_{1}(\ell(\Gamma))$ 和两个实数 $\xi_{1}$ 和 $\xi_{2}$, 均有

$$
\left\|\xi_{1} V_{0}\left(x_{1}\right)+\xi_{2} V_{0}\left(x_{2}\right)\right\|=1 \Longrightarrow \xi_{1} V_{0}\left(x_{1}\right)+\xi_{2} V_{0}\left(x_{2}\right) \in V_{0}\left[S_{1}(\ell(\Gamma))\right] .
$$

证明 注意到子空间

$$
X_{0}=\operatorname{span}\left\{e_{\gamma}: \gamma \in \Gamma\right\}
$$

是稠于空间 $\ell(\Gamma)$ 的. 因此从定理 5 的证明可知, 只需验证下面的条件:

$(\text { ii })^{\prime}$ 对于任意的 $n \in \mathbb{N},\left\{\gamma_{1}, \gamma_{2}, \cdots, \gamma_{n}\right\} \subset \Gamma$, 若存在 $V_{0}(x)=\sum_{k=1}^{n} \xi_{\gamma_{k}} V_{0}\left(e_{\gamma_{k}}\right)$, 则有

$$
x=\sum_{k=1}^{n} \xi_{\gamma_{k}} e_{\gamma_{k}} .
$$

下面, 我们就来验证此结论. 事实上, 由引理 1 可以得到 $V_{0}\left(-e_{\gamma}\right)=-V_{0}\left(e_{\gamma}\right)(\forall \gamma \in \Gamma)$. 类似于 (16) 和 (17) 式, 对于 $\xi_{\gamma_{1}} \neq 0$, 有

和

$$
\left\|V_{0}(x)-\frac{\xi_{\gamma_{1}}}{\left|\xi_{\gamma_{1}}\right|} V_{0}\left(e_{\gamma_{1}}\right)\right\|=2\left(1-\left|\xi_{\gamma_{1}}\right|\right)
$$

$$
\begin{aligned}
\left\|x-\frac{\xi_{\gamma_{1}}}{\left|\xi_{\gamma_{1}}\right|} e_{\gamma_{1}}\right\| & =\left\|\sum_{\gamma} x(\gamma) e_{\gamma}-\frac{\xi_{\gamma_{1}}}{\left|\xi_{\gamma_{1}}\right|} e_{\gamma_{1}}\right\| \\
& =\left\|\left(x\left(\gamma_{1}\right)-\frac{\xi_{\gamma_{1}}}{\left|\xi_{\gamma_{1}}\right|}\right) e_{\gamma_{1}}+\sum_{\gamma \neq \gamma_{1}} x(\gamma) e_{\gamma}\right\|
\end{aligned}
$$




$$
\begin{aligned}
& =\left|x\left(\gamma_{1}\right)-\frac{\xi_{\gamma_{1}}}{\left|\xi_{\gamma_{1}}\right|}\right|+\sum_{\gamma \neq \gamma_{1}}|x(\gamma)| \\
& =\left|x\left(\gamma_{1}\right)-\frac{\xi_{\gamma_{1}}}{\left|\xi_{\gamma_{1}}\right|}\right|+\left(1-\left|x\left(\gamma_{1}\right)\right|\right) \\
& =\left(1-\frac{\xi_{\gamma_{1}}}{\left|\xi_{\gamma_{1}}\right|} x\left(\gamma_{1}\right)\right)+\left(1-\left|x\left(\gamma_{1}\right)\right|\right) \\
& =2-\left(\frac{\xi_{\gamma_{1}}}{\left|\xi_{\gamma_{1}}\right|} x\left(\gamma_{1}\right)+\left|x\left(\gamma_{1}\right)\right|\right) .
\end{aligned}
$$

由于 $V_{0}$ 是 $S_{1}(\ell(\Gamma))$ 上的等距算子, 则从 $(26)$ 和 $(27)$ 式可导出

$$
2\left|\xi_{\gamma_{1}}\right|=\left|x\left(\gamma_{1}\right)\right|+\frac{\xi_{\gamma_{1}}}{\left|\xi_{\gamma_{1}}\right|} x\left(\gamma_{1}\right) .
$$

因此得到 $x\left(\gamma_{1}\right)=\xi_{\gamma_{1}}$.

我们用同样的方法亦可得到 $x\left(\gamma_{k}\right)=\xi_{\gamma_{k}}(2 \leqslant k \leqslant n)$. 并且由引理 3 和 (20) 式, 还有

$$
\sum_{k=1}^{n}\left|x\left(\gamma_{k}\right)\right|=\sum_{k=1}^{n}\left|\xi_{\gamma_{k}}\right|=\left\|\sum_{k=1}^{n} \xi_{\gamma_{k}} V_{0}\left(e_{\gamma_{k}}\right)\right\|=\left\|V_{0}(x)\right\|=1,
$$

因而导出 $x(\gamma)=0\left(\forall \gamma \in \Gamma \backslash\left\{\gamma_{1}, \gamma_{2}, \cdots, \gamma_{n}\right\}\right)$. 推理证毕.

由推理 7 , 我们立即得到文献 [13] 和 [17] 的一个推广命题如下:

推理 8 设 $E$ 是 Banach 空间, $V_{0}$ 是从单位球面 $S_{1}(\ell(\Gamma))$ 到单位球面 $S_{1}(E)$ 上的满等 距算子 (特别地, 如果 $V_{0}$ 是单位球面 $S_{1}(\ell(\Gamma))$ 和 $S_{1}(\ell(\Delta))$ 间的满等距算子), 则 $V_{0}$ 必可以延 拓成全空间 $\ell(\Gamma)$ 上的线性等距算子.

\section{4 进一步的结果}

为改进定理 5 , 需要下面 3 个引理:

引理 9 设 $V_{0}$ 如引理 1 所设, 则

$$
V_{0}(-x)=-V_{0}(x), \quad \forall x \in S_{1}(L(T, \mu)) .
$$

证明 因为 $V_{0}$ 满足引理 1 的假设, 对于任意的元 $x \in S_{1}(L(T, \mu))$, 可知存在元 $\bar{x} \in$ $S_{1}(L(T, \mu))$, 使得

$$
V_{0}(\bar{x})=-V_{0}(x) .
$$

由引理 1 , 对于任意可测子集 $A \subset T(0<\mu(A)<\infty)$, 有

$$
\begin{aligned}
\left\|\bar{x}+\frac{\chi_{A}}{\mu(A)}\right\| & =\left\|\bar{x}-\left(-\frac{\chi_{A}}{\mu(A)}\right)\right\|=\left\|V_{0}(\bar{x})-V_{0}\left(-\frac{\chi_{A}}{\mu(A)}\right)\right\| \\
& =\left\|-V_{0}(x)+V_{0}\left(\frac{\chi_{A}}{\mu(A)}\right)\right\|=\left\|x-\frac{\chi_{A}}{\mu(A)}\right\| .
\end{aligned}
$$

类似地, 同样有

$$
\left\|-\bar{x}+\frac{\chi_{A}}{\mu(A)}\right\|=\left\|x+\frac{\chi_{A}}{\mu(A)}\right\| .
$$

也即得到

$$
\int_{A}\left|\bar{x}(t)+\frac{1}{\mu(A)}\right| \mu(d t)+\int_{A^{c}}|\bar{x}(t)| \mu(d t)=\int_{A}\left|x(t)-\frac{1}{\mu(A)}\right| \mu(d t)+\int_{A^{c}}|x(t)| \mu(d t)
$$


和

$$
\int_{A}\left|\bar{x}(t)-\frac{1}{\mu(A)}\right| \mu(d t)+\int_{A^{c}}|\bar{x}(t)| \mu(d t)=\int_{A}\left|x(t)+\frac{1}{\mu(A)}\right| \mu(d t)+\int_{A^{c}}|x(t)| \mu(d t) .
$$

注意到 $\|\bar{x}\|=\|x\|=1$, 从上面两个等式则可导出

$$
\int_{A}\left(\left|\bar{x}(t)+\frac{1}{\mu(A)}\right|-|\bar{x}(t)|\right) \mu(d t)=\int_{A}\left(\left|x(t)-\frac{1}{\mu(A)}\right|-|x(t)|\right) \mu(d t)
$$

和

$$
\int_{A}\left(\left|\bar{x}(t)-\frac{1}{\mu(A)}\right|-|\bar{x}(t)|\right) \mu(d t)=\int_{A}\left(\left|x(t)+\frac{1}{\mu(A)}\right|-|x(t)|\right) \mu(d t) .
$$

注意到可测集 $A$ 的任意性, 如果 $x(t) \leqslant 0(\forall t \in A)$, 则从 (30) 式, 有

$$
\int_{A}\left(\left|\bar{x}(t)+\frac{1}{\mu(A)}\right|-|\bar{x}(t)|\right) \mu(d t)=1,
$$

这就导出了 $\bar{x}(t) \geqslant 0$, a.e. $t \in A$.

如果 $x(t) \leqslant 0(\forall t \in A)$, 则从 (31) 式, 类似地有 $\bar{x}(t) \leqslant 0$ (a.e. $t \in A)$. 因此综上我们得到

$$
x(t) \cdot \bar{x}(t) \leqslant 0, \quad \text { a.e. } \quad t \in T .
$$

而且从 (30) 和 (31) 式, 我们还可以得到

$$
\int_{A}\left(\left|\bar{x}(t)+\frac{1}{\mu(A)}\right|-\left|\bar{x}(t)-\frac{1}{\mu(A)}\right|\right) \mu(d t)=\int_{A}\left(\left|x(t)-\frac{1}{\mu(A)}\right|-\left|x(t)+\frac{1}{\mu(A)}\right|\right) \mu(d t) .
$$

下面, 我们将断言

$$
\bar{x}(t)=-x(t), \quad \text { a.e. } \quad t \in T .
$$

反之, 假设存在可测子集 $C, \mu(C)>0$, 使得 $\bar{x}(t) \neq-x(t)(\forall t \in C)$. 因为 $x(t), \bar{x}(t) \in L(T, \mu)$, 因此可以找到可测子集 $B \subset C$, 使得 $x(t)$ 和 $\bar{x}(t)$ 在 $B$ 上有界, 且对于测度充分小的可测子 集 $A \subset B$, 或有 $x(t) \geqslant 0$, 或有 $x(t) \leqslant 0(\forall t \in A)$. 而由 (32) 式可知: 如果 $x(t) \geqslant 0(\forall t \in A)$, 则 $\bar{x}(t) \leqslant 0(\forall t \in A)$, 因此我们导出

$$
\begin{aligned}
\int_{A}(\mid \bar{x}(t) & \left.+\frac{1}{\mu(A)}|-| \bar{x}(t)-\frac{1}{\mu(A)} \mid\right) \mu(d t) \\
& =\int_{A}\left(\left|\frac{1}{\mu(A)}-(-\bar{x}(t))\right|-\left|\frac{1}{\mu(A)}+(-\bar{x}(t))\right|\right) \mu(d t) \\
& =-2 \int_{A} \min \left(-\bar{x}(t), \frac{1}{\mu(A)}\right) \mu(d t)=2 \int_{A} \bar{x}(t) \mu(d t)
\end{aligned}
$$

和

$$
\begin{aligned}
& \int_{A}\left(\left|x(t)+\frac{1}{\mu(A)}\right|-\left|x(t)-\frac{1}{\mu(A)}\right|\right) \mu(d t) \\
& \quad=-2 \int_{A} \min \left(x(t), \frac{1}{\mu(A)}\right) \mu(d t)=2 \int_{A} x(t) \mu(d t),
\end{aligned}
$$

也即

$$
\int_{A}\left(\left|\bar{x}(t)+\frac{1}{\mu(A)}\right|-\left|\bar{x}(t)-\frac{1}{\mu(A)}\right|\right) \mu(d t) \neq \int_{A}\left(\left|x(t)-\frac{1}{\mu(A)}\right|-\left|x(t)+\frac{1}{\mu(A)}\right|\right) \mu(d t) .
$$

类似地, 如果 $x(t) \leqslant 0(\forall t \in A)$, 我们同样可以导出以上结论. 但此显然与 (33) 式矛盾. 证毕.

注 10 我们不用引理 1 也可以证得引理 9 , 但需要引理 1 的证明技巧. 下面给出粗略的 证明. 
首先, 令 $A_{+}=\{t: \bar{x}(t) \cdot x(t)>0\}$ 及 $A_{-}=\{t: \bar{x}(t) \cdot x(t) \leqslant 0\}$. 应用等式

$$
2=\left\|-2 V_{0}(x)\right\|=\left\|V_{0}(\bar{x})-V_{0}(x)\right\|=\|\bar{x}-x\|,
$$

有 $\bar{x}(t) \cdot x(t) \leqslant 0$ (a.e. $t \in T)$.

其次, 类似于 (5) 式的定义, 对于任意可测集 $B$, 其满足 $0<\mu(B)<\infty$ 和 $B \cap A=\emptyset(A=$ $\operatorname{supp} x)$, 假设 $V_{0}\left(\bar{y}_{ \pm}\right)=-V_{0}\left(\frac{1}{2} x \pm \frac{1}{2} \frac{\chi_{B}}{\mu(B)}\right)$, 则可以得到与 $(7)$ 式类似的结论: $\bar{y}_{ \pm}(t) \cdot x(t) \leqslant 0$ (a.e. $t \in A$ ). 由此则有

$$
\begin{aligned}
2 & =\left\|\bar{y}_{ \pm}-x\right\|=\left\|V_{0}\left(\bar{y}_{ \pm}\right)-V_{0}(x)\right\| \\
& =\left\|-V_{0}\left(\frac{1}{2} x \pm \frac{1}{2} \frac{\chi_{B}}{\mu(B)}\right)+V_{0}(\bar{x})\right\|=\left\|\bar{x}-\left(\frac{1}{2} x \pm \frac{1}{2} \frac{\chi_{B}}{\mu(B)}\right)\right\|,
\end{aligned}
$$

从而导出了

$$
\frac{1}{2}=\int_{B}\left|\bar{x}(t) \mp \frac{1}{2 \mu(B)}\right| \mu(d t)-\int_{B}|\bar{x}(t)| \mu(d t) .
$$

这样一来, 我们可以得到 $\bar{x}(t)=0$, a.e. $t \in A^{c}$.

最后, 令 $V_{0}\left(\bar{x}_{0}\right)=-V_{0}\left(\frac{\left.x\right|_{A_{0}}}{\left\|\left.x\right|_{A_{0}}\right\|}\right)\left(\forall A_{0} \subset A, \mu\left(A_{0}\right)>0\right)$. 由下面不等式

$$
\begin{aligned}
2\left(1-\left\|\left.x\right|_{A_{0}}\right\|\right) & =\left\|\frac{\left.x\right|_{A_{0}}}{\left\|\left.x\right|_{A_{0}}\right\|}-x\right\|=\left\|-V_{0}(x)-V_{0}\left(\bar{x}_{0}\right)\right\| \\
& =\left\|V_{0}(\bar{x})-V_{0}\left(\bar{x}_{0}\right)\right\|=\left\|\bar{x}-\bar{x}_{0}\right\| \\
& \geqslant 2\left(1-\int_{A_{0}}|\bar{x}(t)| \mu(d t)\right),
\end{aligned}
$$

我们有

$$
\int_{A_{0}}(|\bar{x}(t)|-|x(t)|) \mu(d t) \geqslant 0 .
$$

注意到 $\|x\|=\|\bar{x}\|=1$ 和 $A_{0}$ 的任意性, 便可导出 $\bar{x}(t)=-x(t)$ (a.e. $t \in T$ ). 因此再次完成了 定理 9 的证明.

引理 11 设 $V_{0}$ 如引理 1 所设, 如果

$$
V_{0}(x)=\xi_{1} V_{0}\left(x_{1}\right)+\xi_{2} V_{0}\left(x_{2}\right)
$$

(其中, $\xi_{i} \neq 0(i=1,2), x_{1}, x_{2} \in S_{1}(L(T, \mu))$ 且 $\operatorname{supp} x_{1} \cap \operatorname{supp} x_{2}=\emptyset$ ), 则有

$$
x=\xi_{1} x_{1}+\xi_{2} x_{2} .
$$

证明 设 $A_{1}=\operatorname{supp} x_{1}$ 及 $A_{2}=\operatorname{supp} x_{2}$, 从假设有 $A_{1} \cap A_{2}=\emptyset$. 由于证明过程较长, 下 面我们分三步进行.

步骤 I 我们将断言: $\operatorname{supp} x \subset A_{1} \cup A_{2}, \int_{A_{i}}|x(t)| \mu(d t)=\left|\xi_{i}\right|$ 及 $x(t) \cdot \xi_{i} x_{i}(t) \geqslant 0, \forall t \in$ $A_{i}(i=1,2)$.

事实上, 从引理 3 和 9 , 我们有

$$
\begin{aligned}
\left\|V_{0}(x)-\frac{\xi_{1}}{\left|\xi_{1}\right|} V_{0}\left(x_{1}\right)\right\| & =\left\|-\frac{\xi_{1}}{\left|\xi_{1}\right|}\left(1-\left|\xi_{1}\right|\right) V_{0}\left(x_{1}\right)+\xi_{2} V_{0}\left(x_{2}\right)\right\| \\
& =\left\|-\frac{\xi_{1}}{\left|\xi_{1}\right|}\left|\xi_{2}\right| V_{0}\left(x_{1}\right)+\xi_{2} V_{0}\left(x_{2}\right)\right\| \\
& =\left|\xi_{2}\right|+\left|\xi_{2}\right|=2\left|\xi_{2}\right| .
\end{aligned}
$$


另一方面, 注意到 $\|x\|=1$, 又有

$$
\begin{aligned}
\| x-\frac{\xi_{1}}{\left|\xi_{1}\right|} x_{1} \mid & =\int_{A_{1}}\left|x(t)-\frac{\xi_{1} x_{1}(t)}{\left|\xi_{1}\right|}\right| \mu(d t)+\int_{A_{1}^{c}}|x(t)| \mu(d t) \\
& =\int_{A_{1}}\left|x(t)-\frac{\xi_{1} x_{1}(t)}{\left|\xi_{1}\right|}\right| \mu(d t)+\left(1-\int_{A_{1}}|x(t)| \mu(d t)\right) .
\end{aligned}
$$

现令

$$
A_{1}^{+}=\left\{t \in A_{1}: x(t) \cdot \xi_{1} x_{1}(t) \geqslant 0\right\}, \quad A_{1}^{-}=\left\{t \in A_{1}: x(t) \cdot \xi_{1} x_{1}(t)<0\right\} .
$$

如果 $\xi_{1}>0$, 那么从 $x_{1}$ 和 $A_{1}$ 的假设得到

$$
\begin{aligned}
\int_{A_{1}}\left(\left|x(t)-\frac{\xi_{1}}{\left|\xi_{1}\right|} x_{1}(t)\right|-|x(t)|\right) \mu(d t) \\
\quad=\int_{A_{1}^{+}}\left(\left|x(t)-x_{1}(t)\right|-|x(t)|\right) \mu(d t)+\int_{A_{1}^{-}}\left|x_{1}(t)\right| \mu(d t) \\
\quad=\int_{A_{1}^{+}}\left(|| x(t)|-| x_{1}(t)||-|x(t)|\right) \mu(d t)+\left(1-\int_{A_{1}^{+}}\left|x_{1}(t)\right| \mu(d t)\right) .
\end{aligned}
$$

由于 $V_{0}$ 是等距算子, 从引理 9 及 (35)-(37) 式可以导出

$$
\begin{aligned}
2\left|\xi_{1}\right| & =2\left(1-\left|\xi_{2}\right|\right)=\int_{A_{1}^{+}}\left(|x(t)|+\left|x_{1}(t)\right|-|| x(t)|-| x_{1}(t)||\right) \mu(d t) \\
& =2 \int_{A_{1}^{+}} \min \left(|x(t)|,\left|x_{1}(t)\right|\right) \mu(d t) \leqslant 2 \int_{A_{1}^{+}}|x(t)| \mu(d t) .
\end{aligned}
$$

也即得到

$$
\left|\xi_{1}\right| \leqslant \int_{A_{1}^{+}}|x(t)| \mu(d t) .
$$

如果 $\xi_{1}<0$, 类似地讨论也有 $(38)$ 式. 而用相同的方法, 亦可得到

$$
\left|\xi_{2}\right| \leqslant \int_{A_{2}^{+}}|x(t)| \mu(d t) \text {. }
$$

再次应用引理 9 和 3 , 则有

$$
1=\left\|V_{0}(x)\right\|=\left|\xi_{1}\right|+\left|\xi_{2}\right|=\int_{T}|x(t)| \mu(d t),
$$

这样一来, 从上式可知 (38) 和 (39) 式就证明了步骤 I.

步骤 II. 我们将证明

$$
\mu\left(\left\{t \in A_{i}:|x(t)|>\left|x_{i}(t)\right|\right\}\right)=0, \quad i=1,2 .
$$

下面, 不失一般性, 只需对子集 $A_{1}$ 来证明结论. 令

$$
B_{1}=\left\{t \in A_{1}:|x(t)|>\left|x_{1}(t)\right|\right\}, \quad S_{1}=\left\{t \in A_{1}:|x(t)| \leqslant\left|x_{1}(t)\right|\right\} .
$$

由步骤 I 和 $\operatorname{supp} x_{1}=A_{1}$, 有

$$
\begin{aligned}
\left\|x-\frac{\xi_{1}}{\left|\xi_{1}\right|} x_{1}\right\| & =\int_{A_{1}}\left|x(t)-\frac{\xi_{1}}{\left|\xi_{1}\right|} x_{1}(t)\right| \mu(d t)+\int_{A_{2}}|x(t)| \mu(d t) \\
& =\int_{A_{1}}|| x(t)|-| x_{1}(t)|| \mu(d t)+\left|\xi_{2}\right| \\
& =\int_{B_{1}}\left(|x(t)|-\left|x_{1}(t)\right|\right) \mu(d t)+\int_{S_{1}}\left(\left|x_{1}(t)\right|-|x(t)|\right) \mu(d t)+\left|\xi_{2}\right|
\end{aligned}
$$




$$
\begin{aligned}
& =\int_{B_{1}}\left(|x(t)|-\left|x_{1}(t)\right|\right) \mu(d t)+\left(1-\int_{B_{1}}\left|x_{1}(t)\right| \mu(d t)\right)-\left(\left|\xi_{1}\right|-\int_{B_{1}}|x(t)| \mu(d t)\right)+\left|\xi_{2}\right| \\
& =2\left|\xi_{2}\right|-2 \int_{B_{1}}\left(\left|x_{1}(t)\right|-|x(t)|\right) \mu(d t),
\end{aligned}
$$

再次注意到 $V_{0}$ 是等距算子, 从 (35) 式及应用引理 9 , 则可得到

$$
2\left|\xi_{2}\right|=2\left|\xi_{2}\right|-2 \int_{B_{1}}\left(\left|x_{1}(t)\right|-|x(t)|\right) \mu(d t),
$$

也即

$$
\int_{B_{1}}\left(\left|x_{1}(t)\right|-|x(t)|\right) \mu(d t)=0 .
$$

最后, 从集合 $B_{1}$ 的定义和 (41) 式立即可以导出 $\mu\left(B_{1}\right)=0$, 这就证明了步骤 II.

步骤 III. 我们将证明 $x(t)=\xi_{1} x_{1}(t)+\xi_{2} x_{2}(t)$.

不失一般性, 只需证明

$$
x(t)=\xi_{1} x_{1}(t), \quad \text { a.e. } \quad t \in A_{1} .
$$

对于任意可测集 $A_{1,0} \subset A_{1}$ 且 $\mu\left(A_{1,0}\right)>0$, 从步骤 I 和 II 及 $\left\|\left.x_{1}\right|_{A_{1,0}}\right\| \leqslant 1$, 可以导出

$$
\begin{aligned}
\left\|\frac{\xi_{1} x_{1} \mid A_{1,0}}{\left\|\xi_{1} x_{1} \mid A_{1,0}\right\|}-x\right\| & =\int_{A_{1,0}}\left|\frac{\xi_{1} x_{1}(t)}{\left|\xi_{1}\right|\left\|x_{1} \mid A_{1,0}\right\|}-x(t)\right| \mu(d t)+\int_{A_{1,0}^{c}}|x(t)| \mu(d t) \\
& =\int_{A_{1,0}}\left(\frac{\left|x_{1}(t)\right|}{\left\|x_{1} \mid A_{1,0}\right\|}-|x(t)|\right) \mu(d t)+\left(1-\int_{A_{1,0}}|x(t)| \mu(d t)\right) \\
& =2\left(1-\int_{A_{1,0}}|x(t)| \mu(d t)\right) .
\end{aligned}
$$

另一方面, 由引理 $9 、 x_{1}$ 和 $x_{2}$ 支撑不交的假设, 又有

$$
\begin{aligned}
& \left\|V_{0}(x)-V_{0}\left(\frac{\left.\xi_{1} x_{1}\right|_{A_{1,0}}}{\left|\xi_{1}\right|\left\|\left.x_{1}\right|_{A_{1,0}}\right\|}\right)\right\| \\
& =\left\|\left(\xi_{1} V_{0}\left(x_{1}\right)+\xi_{2} V_{0}\left(x_{2}\right)\right)-V_{0}\left(\frac{\xi_{1}}{\left|\xi_{1}\right|} \frac{\left.x_{1}\right|_{A_{1,0}}}{\left\|\left.x_{1}\right|_{A_{1,0}}\right\|}\right)\right\| \\
& =\left\|\left|\xi_{1}\right|\left[V_{0}\left(\frac{\xi_{1}}{\left|\xi_{1}\right|} x_{1}\right)-V_{0}\left(\frac{\xi_{1}}{\left|\xi_{1}\right|} \frac{x_{1}||_{A_{1,0}}}{\| x_{1}||_{A_{1,0}} \mid}\right)\right]+\left|\xi_{2}\right|\left[V_{0}\left(\frac{\xi_{2}}{\left|\xi_{2}\right|} x_{2}\right)-V_{0}\left(\frac{\xi_{1}}{\left|\xi_{1}\right|} \frac{x_{1}||_{A_{1,0}}}{\left\|\left.x_{1}\right|_{A_{1,0}}\right\|}\right)\right]\right\| \\
& \leqslant\left|\xi_{1}\right|\left\|\frac{\xi_{1}}{\left|\xi_{1}\right|} x_{1}-\frac{\xi_{1}}{\left|\xi_{1}\right|} \frac{x_{1} \mid A_{A_{1,0}}}{\left\|x_{1} \mid A_{A_{1,0}}\right\|}\right\|+\left|\xi_{2}\right|\left\|\frac{\xi_{2}}{\left|\xi_{2}\right|} x_{2}-\frac{\xi_{1}}{\left|\xi_{1}\right|} \frac{\left.x_{1}\right|_{A_{1,0}}}{\left\|\left.x_{1}\right|_{A_{1,0}}\right\|}\right\| \\
& =\left|\xi_{1}\right|\left\|x_{1}-\frac{\left.x_{1}\right|_{A_{1,0}}}{\left\|x_{1} \mid A_{A_{1,0}}\right\|}\right\|+2\left|\xi_{2}\right| \\
& =2\left|\xi_{1}\right|\left(1-\left\|\left.x_{1}\right|_{A_{1,0}}\right\|\right)+2\left|\xi_{2}\right| \\
& =2\left(1-\int_{A_{1,0}}\left|\xi_{1} x_{1}(t)\right| \mu(d t)\right) .
\end{aligned}
$$

因此, 从 (43) 和 (44) 式可以导出

$$
\int_{A_{1,0}}\left(|x(t)|-\left|\xi_{1} x_{1}(t)\right|\right) \mu(d t) \geqslant 0,
$$

及

$$
|x(t)| \geqslant\left|\xi_{1} x_{1}(t)\right|, \quad \text { a.e. } t \in A_{1} .
$$


最后注意到 $A_{1,0}$ 是 $A_{1}$ 的任意子集, 因此从步骤 I 的结果

$$
\int_{A_{1}}|x(t)| \mu(d t)=\int_{A_{1}}\left|\xi_{1} x_{1}(t)\right| \mu(d t)
$$

和 (45) 式, 便可导出所需的 (42) 式. 证毕.

应用引理 11 我们容易证得下面的引理:

引理 12 设 $E$ 是 Banach 空间, $V_{0}$ 是从单位球面 $S_{1}(L(T, \mu))$ 到单位球面 $S_{1}(E)$ 内的 等距算子. 假设对于任意支撑不交的两个元 $x_{1}, x_{2} \in S_{1}(L(T, \mu))$ 和 $\xi_{1}, \xi_{2} \in \mathbb{R}$, 均有

$$
\left\|\xi_{1} V_{0}\left(x_{1}\right)+\xi_{2} V_{0}\left(x_{2}\right)\right\|=1 \Longrightarrow \xi_{1} V_{0}\left(x_{1}\right)+\xi_{2} V_{0}\left(x_{2}\right) \in V_{0}[S(L(T, \mu))] .
$$

则对于任意 $n$ 个相互不交的元 $\left\{x_{1}, x_{2}, \ldots, x_{n}\right\} \subset S_{1}(L(T, \mu))$, 如有

$$
V_{0}(x)=\sum_{k=1}^{n} \xi_{k} V_{0}\left(x_{k}\right),
$$

那么就有

$$
x=\sum_{k=1}^{n} \xi_{k} x_{k} .
$$

证明 对于 $n=2$, 由引理 11 可知结论显然成立. 假设当 $n=m-1$ 时结论成立, 则当 $n=m$ 时, 注意到等式

$$
V_{0}(x)=\left(\left|\xi_{1}\right|+\left|\xi_{2}\right|\right)\left(\frac{\xi_{1}}{\left|\xi_{1}\right|+\left|\xi_{2}\right|} V_{0}\left(x_{1}\right)+\frac{\xi_{2}}{\left|\xi_{1}\right|+\left|\xi_{2}\right|} V_{0}\left(x_{2}\right)\right)+\sum_{k=3}^{m} \xi_{k} V_{0}\left(x_{k}\right) .
$$

故由引理 9 和 3 可以得到

$$
\left\|\frac{\xi_{1}}{\left|\xi_{1}\right|+\left|\xi_{2}\right|} V_{0}\left(x_{1}\right)+\frac{\xi_{2}}{\left|\xi_{1}\right|+\left|\xi_{2}\right|} V_{0}\left(x_{2}\right)\right\|=\frac{\left|\xi_{1}\right|}{\left|\xi_{1}\right|+\left|\xi_{2}\right|}+\frac{\left|\xi_{2}\right|}{\left|\xi_{1}\right|+\left|\xi_{2}\right|}=1,
$$

从而再由假设和引理 11 , 有

$$
\frac{\xi_{1}}{\left|\xi_{1}\right|+\left|\xi_{2}\right|} V_{0}\left(x_{1}\right)+\frac{\xi_{2}}{\left|\xi_{1}\right|+\left|\xi_{2}\right|} V_{0}\left(x_{2}\right)=V_{0}\left(\frac{\xi_{1}}{\left|\xi_{1}\right|+\left|\xi_{2}\right|} x_{1}+\frac{\xi_{2}}{\left|\xi_{1}\right|+\left|\xi_{2}\right|} x_{2}\right) .
$$

因此从归纳假设及 (46) 和 (47) 式导出

$$
\begin{aligned}
x & =\left(\left|\xi_{1}\right|+\left|\xi_{2}\right|\right)\left(\frac{\xi_{1}}{\left|\xi_{1}\right|+\left|\xi_{2}\right|} x_{1}+\frac{\xi_{2}}{\left|\xi_{1}\right|+\left|\xi_{2}\right|} x_{2}\right)+\sum_{k=3}^{m} \xi_{k} x_{k} \\
& =\sum_{k=1}^{m} \xi_{k} x_{k},
\end{aligned}
$$

这样就完成了引理的证明.

从定理 5 和引理 12 , 我们立即得到下面的

定理 13 设 $E$ 是 Banach 空间, $V_{0}$ 是从单位球面 $S_{1}(L(T, \mu))$ 到单位球面 $S_{1}(E)$ 内的 等距算子, 则 $V_{0}$ 能够延拓成全空间 $L(T, \mu)$ 上的 (实) 线性等距算子的充要条件是:

对于任意两个元 $x_{1}, x_{2} \in S_{1}(L(T, \mu))$, 及两个数 $\xi_{1}, \xi_{2} \in \mathbb{R}$, 均有

$$
\left\|\xi_{1} V_{0}\left(x_{1}\right)+\xi_{2} V_{0}\left(x_{2}\right)\right\|=1 \Longrightarrow \xi_{1} V_{0}\left(x_{1}\right)+\xi_{2} V_{0}\left(x_{2}\right) \in V_{0}[S(L(T, \mu))] .
$$

推理 14 设 $E$ 是 Banach 空间, $V_{0}$ 是从单位球面 $S_{1}(L(T, \mu))$ 到单位球面 $S_{1}(E)$ 上 的满等距算子, 则 $V_{0}$ 可以延拓成全空间 $L(T, \mu)$ 上的 (实) 线性等距算子. 特别地, 单位球面 $S_{1}(L(T, \mu))$ 和 $S_{1}(L(S, \nu))$ 间的满等距算子均能延拓为全空间上的线性等距算子. 
1 Tingley D. Isometries of the unit spheres. Geom Dedicata, 22: 371-378 (1987)

2 定光桂. 空间 $\ell^{p}(\Gamma)(p>1)$ 的单位球面间等距算子的延拓. 中国科学 A 辑: 数学, 32(11): 991-995 (2002)

3 Ding G G. The representation theorem of onto isometric mapping between two unit spheres of $\ell^{1}(\Gamma)$ type spaces and the application on isometric extension problem. Acta Math Sin (Engl Ser), 20(6): 1089-1094 (2004)

4 定光桂. 两个 $\ell^{\infty}$ - 型空间单位球面满等距映射的表现理论及其在等距延拓问题上的应用. 中国科学 $\mathrm{A}$ 辑: 数学, 34(2): 157-164 (2004)

5 Wang J. On extension of isometries between unit spheres of $A L_{p}$-spaces $(1<p<\infty)$. Proc Amer Math Soc, 132(10): 2899-2909 (2004)

6 An G M. Isometries on unit sphere of $\left(\ell^{\beta_{n}}\right)$. J Math Anal Appl, 301: 249-254 (2005)

7 Fu X H. Isometries on the space s. Acta Math Sci Ser B Engl Ed, 26(3): 502-508 (2006)

8 Yang X Z. On extension of isometries between unit spheres of $L_{p}(\mu)$ and $L_{p}(\nu, H)(1<p \neq 2, H$ is a Hilbert space). J Math Anal Appl, 323: 985-992 (2006)

9 Ding G G. On the extension of isometries between unit spheres of $E$ and $C(\Omega)$. Acta Math Sin (Engl Ser), 19(4): 793-800 (2003)

10 Ding G G. The 1-Lipschitz mapping between the unit spheres of two Hilbert spaces can be extended to a real linear linear isometry of the whole space. Sci China Ser A-Math, 45(4): 479-483 (2002)

11 Hou Z B, Zhang L J. The isometric extension of the into mapping between the unit spheres of $A L_{p^{-}}$ spaces $(1<p<\infty)$. Acta Math Sinica (Chin Ser), 50(6): 1435-1440 (2007)

12 Ding G G. The isometric extension of the into mapping from the unit sphere $S\left(\ell_{(2)}^{\infty}\right)$ to $S\left(L^{1}(\mu)\right)$. Acta Math Sin (Engl Ser), 22(6): 1721-1724 (2006)

13 Ding G G. The isometric extension of the into mapping from the $\mathcal{L}^{\infty}(\Gamma)$-type space to some normed space E. Illinois J Math, 52(2): 445-453 (2007)

14 Fang X N, Wang J H. On extension of isometries between the unit spheres of normed soace $E$ and $C(\Omega)$. Acta Math Sin (Engl Ser), 22(6): 1819-1824 (2006)

$15 \mathrm{Fu} \mathrm{X} \mathrm{H.} \mathrm{The} \mathrm{isometric} \mathrm{extension} \mathrm{of} \mathrm{the} \mathrm{into} \mathrm{mapping} \mathrm{from} \mathrm{unit} \mathrm{sphere} S_{1}(E)$ to $S_{1}\left(\ell^{\infty}(\Gamma)\right)$. Acta Math Sin (Engl Ser), to appear, 2008

16 Liu R. On extension of isometries between the unit sphere oof $\mathcal{L}^{\infty}$-type space and some Banach space $E$. J Math Anal Appl, 333: 959-970 (2007)

17 Ding G G. The isometric extension of the into mapping from the unit sphere $S(\ell(\Gamma))$ to $S(E)$. Acta Math Sci, to appear, 2008

18 Lindenstrauss J, Tzafriri L. Classical Banach Spaces II. Berlin-Heildelberg-New York: Springer-Verlag, 1979 